\title{
Assembly of Superparamagnetic Filaments in External Field
}

Article in Langmuir · August 2016

DOI: 10.1021/acs.langmuir.6b02268

CITATIONS

0

3 authors, including:

\section{Jason Wei}

Chinese Academy of Sciences

18 PUBLICATIONS 4 CITATIONS

SEE PROFILE
READS

45

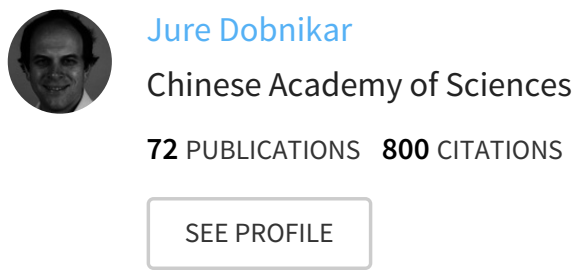




\section{Assembly of Superparamagnetic Filaments in External Field}

Jiachen Wei, ${ }^{, \dagger, \$}$ Fan Song, ${ }^{\dagger, \ddagger}$ and Jure Dobnikar, ${ }^{*}, \mathbb{I l}$,

†State Key Laboratory of Nonlinear Mechanics, Institute of Mechanics, Chinese Academy of Sciences, 15 Beisihuanxi Road, Beijing 100190, China

${ }^{\ddagger}$ School of Engineering Science, University of Chinese Academy of Sciences, Beijing 100049, China

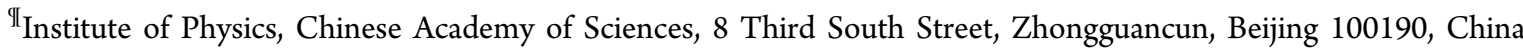

${ }^{\S}$ International Research Center for Soft Matter, Beijing University of Chemical Technology, 15 Beisanhuan Road, Beijing 100029, China

\section{Supporting Information}

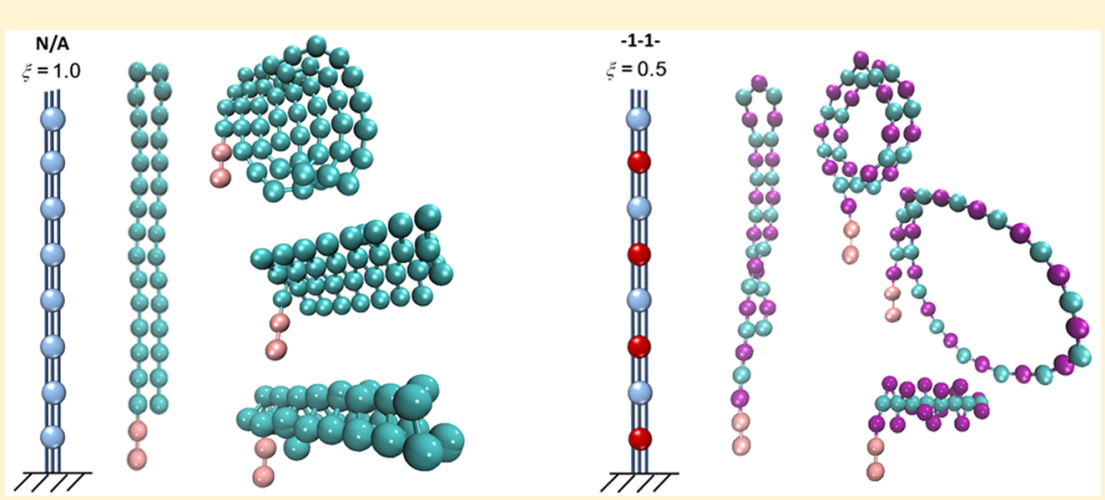

ABSTRACT: We present a theoretical and simulation study of anchored magneto-elastic filaments in external magnetic field. The filaments are composed of a mixture of superparamagnetic and nonmagnetic colloidal beads interlinked with elastic springs. We explore the steady-state structures of filaments with various composition and bending rigidity subject to external magnetic field parallel to the surface. The interplay of elastic and induced magnetic interactions results in a rich phase behavior with morphologies reminiscent of macromolecular folding: bent filaments, loops, sheets, helicoids, and other collapsed structures. Our results provide new insights into the design of hierarchically assembled supramolecular structures with controlled response to external stimuli.

\section{INTRODUCTION}

Thin films with complex architecture and controlled response to external stimuli are promising systems for designing responsive functional coatings. Polymer and polyelectrolyte brushes have been extensively explored in this context. ${ }^{1-4}$ Their swelling and collapse can be externally manipulated by electric fields, temperature, $\mathrm{pH}$, or pressure. Specific patterns can form upon loading the brushes with colloids or nanoparticles ${ }^{5,6}$ as a result of internal competing interactions. ${ }^{7-15}$ To date much less explored are magnetoresponsive coatings. ${ }^{16-18}$ Theoretically, the properties of magnetic filaments, i.e., chains of magnetic nanoparticles linked with elastic linkers, under external driving have been explored in bulk ${ }^{19-25}$ where they have been shown to bend into "S"-like or "U"-like shapes. ${ }^{19}$ The bending threshold of an anchored magnetoelastic rod has recently been determined experimentally and theoretically. ${ }^{13}$ Soft superparamagnetic filaments have been exploited in designing artificial swimmers ${ }^{24,26-29}$ and biomedical devices, ${ }^{30-32}$ and more rigid structures with ferromagnetic or superparamagnetic nanoparticles find applications in cellular engineering ${ }^{12,13}$ and designing advanced materials such as biomimetic cilia ${ }^{33,34}$ and micromechanical sensors. ${ }^{20-23,25}$ In recent simulations, ${ }^{35}$ supracolloidal filaments composed of ferromagnetic colloidal beads linked via cross-linked polymers and grafted to a surface have been explored as a realization of magnetoresponsive surface coatings.

Here we study the structural properties and phase behavior of isolated anchored superparamagnetic filaments in constant external magnetic field parallel to the surface. The model filaments are composed of neutral and superparamagnetic nanocolloidal beads interlinked with elastic bonds (see Figure 1). We perform molecular dynamics (MD) simulations and energy minimization (EM) and study how the morphology of filaments is governed by their composition and by the interplay of elastic and magnetic interactions. Superparamagnetic colloids acquire a magnetic moment in the direction of the externally applied field and thus tend to bend if a strong enough field is applied in the direction parallel to the surface. We determine

Received: June 20, 2016

Revised: August 17, 2016 


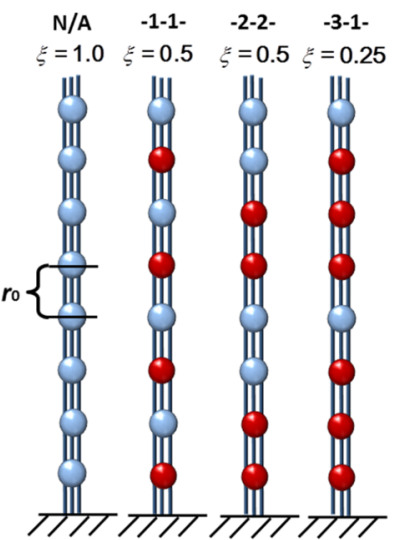

(a)

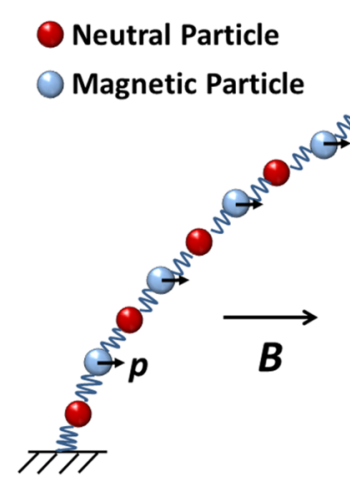

(b)
Figure 1. (a) Sketch of two types of models implemented in our simulation. While $\xi=1.0$ represents exclusive magnetic filament, any giving $\xi<1.0$ denotes neutral-magnetic combined filament of different unit sequence. Here we have, from left to right, " $-1-1-$ ", "$2-2-$ ", and " $-3-1-$ " sequences for $\xi<1.0$ cases. The blue and red spheres respectively represent the magnetic and neutral beads. (b) Bending of a neutral-magnetic combined filament in constant external field $\mathbf{B}$.

the bending line by performing EM and find an agreement with the experiments on clamped superparamagnetic rods. ${ }^{13}$ In $\mathrm{MD}$ simulations within the bending regime we observe controllable self-assembled patterns such as loops, sheets, and collapsed clusters. Different from the study of Sanchez et al., ${ }^{35}$ we explore a wide range of elastic parameters including very flexible filaments, where the morphologies are much richer than in the case of stiff magnetoelastic polymers. Moreover, we study the case with magnetic field acting in-plane-resulting in a frustration between elastic and magnetic interaction and in emergence of new complex structures.

The artcle is organized as follows: the next two sections introduce the model and simulation methods, respectively. Next, the determination of the phase boundaries and the analysis of structural properties of anchored magnetic filaments are presented. The last section is the summary.

\section{MODEL}

We model superparamagnetic filament as a chain of colloidal beads that are either nonmagnetic or superparamagnetic and are linked by harmonic bonds. The chain is anchored to a hard neutral surface; see Figure 1. The composition of each filament is characterized by the number of the beads $L$, the fraction of magnetic beads $\xi=L_{\mathrm{m}} / L$, and the specific sequence of magnetic and nonmagnetic beads. For relaxed filaments without the presence of the magnetic field, $L$ is proportional to their length. Under the action of the magnetic field, the length of the filaments depends on the magnetic forces as well; however, in the stretching rigidity regime we explore, this effect is minor. We study filaments with periodic sequences with repeat units of $N_{\mathrm{n}}$ nonmagnetic beads followed by $N_{\mathrm{m}}$ magnetic ones (the fraction $\xi$ is then $\left.\xi=N_{\mathrm{m}} /\left(N_{\mathrm{m}}+N_{\mathrm{n}}\right)\right)$ and denote them as "$N_{\mathrm{n}}-N_{\mathrm{m}}-$.

The interaction between beads is modeled by a repulsive Weeks-Chandler-Andersen (WCA) potential (truncated and shifted Lennard-Jones interaction)

$$
U_{i j}^{\mathrm{WCA}}=\left\{\begin{array}{c}
4 \epsilon\left[\left(\frac{\sigma}{r_{i j}}\right)^{12}-\left(\frac{\sigma}{r_{i j}}\right)^{6}\right]+\epsilon, r_{i j} \leqslant 2^{1 / 6} \sigma \\
0, r_{i j}>2^{1 / 6} \sigma
\end{array}\right.
$$

where $\epsilon$ is the magnitude of the interaction, $\sigma$ the diameter (in our reduced units, $\epsilon=\sigma=1$, see Table 1 ), and $r_{i j}$ is the distance

Table 1. Table of Reduced Units

\begin{tabular}{ll}
\multicolumn{1}{c}{ quantity } & reduced unit \\
distance & $\sigma$ \\
magnetic field & $12 \sqrt{\frac{\epsilon \mu_{0}}{\chi_{\mathrm{m}}^{2} \pi \sigma^{3}}}$ \\
magnetic moment & $\sqrt{\frac{4 \pi \sigma^{3} \epsilon}{\mu_{0}}}$ \\
energy & $\epsilon$ \\
temperature & $\epsilon / k_{\mathrm{B}}$ \\
mass & $M$ \\
time & $\sqrt{\frac{M \sigma^{2}}{\epsilon}}$
\end{tabular}

between two beads. Besides the WCA repulsion, the beads are linked with bonded interactions, including the stretching $U^{r}$, bending $U^{\theta}$, and twisting $U^{\phi}$ parts

$$
U_{i}^{r}=\frac{1}{2} K_{r}\left(r_{i, i+1}-r_{0}\right)^{2}, \quad U_{i}^{\theta}=\frac{1}{2} K_{\theta} \theta_{i}^{2}, \quad U_{i}^{\phi}=\frac{1}{2} K_{\phi} \phi_{i}^{2}
$$

where $r_{0}$ is the equilibrium bond distance, $K_{r}$ is the linear stiffness of the bond, $K_{\theta}$ the bending, and $K_{\phi}$ the twist rigidity. The bending angle $\theta$ is defined for three consecutive beads, while $\phi$ is the improper dihedral angle defined for four consecutive beads. ${ }^{36}$ The first bead in the filament is anchored to the surface with the equilibrium direction pointing perpendicularly to the surface; therefore, in the absence of external magnetic field, the filaments are on average straight and perpendicular to the surface. Together with the WCA interactions (eq 1) the elastic terms (eq 2) constitute the bonding interactions between the beads in the filaments. Under the action of external magnetic field, the magnetic colloids acquire magnetic moments, which lead to additional dipoledipole interactions

$$
U_{i j}^{\mathrm{pp}}=\frac{\mu_{0}}{4 \pi}\left[\frac{1}{r_{i j}^{3}}\left(\mathbf{p}_{i} \cdot \mathbf{p}_{j}\right)-\frac{3}{r_{i j}^{5}}\left(\mathbf{p}_{i} \cdot \mathbf{r}_{i j}\right)\left(\mathbf{p}_{j} \cdot \mathbf{r}_{i j}\right)\right]
$$

where $\mathbf{p}_{i}$ and $\mathbf{p}_{j}$ respectively represent the dipole moment of particles $i$ and $j$. The units of $\mathbf{p}$ and $\mathbf{B}$ are chosen (Table 1) so that the reduced dipole moment is equal to the applied field strength. Since the external field is constant and we only consider a small number of magnetic beads, we disregard manybody depolarizing forces among them. This simplification amounts to an error less than $1-2 \%$ in the magnetic interaction energy and thus does not qualitatively affect the equilibrium structures of the filaments. Throughout the work and in the above expressions (eqs 1-3), we use reduced units that are summarized in the Table 1.

The total energy of the system is obtained as a sum

$$
U=\sum_{i<j}\left(U_{i j}^{\mathrm{WCA}}+U_{i j}^{\mathrm{pp}}\right)+\sum_{i}\left(U_{i}^{r}+U_{i}^{\theta}+U_{i}^{\phi}\right)
$$




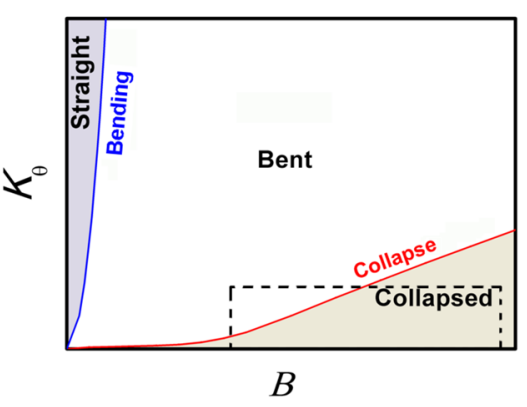

(a)

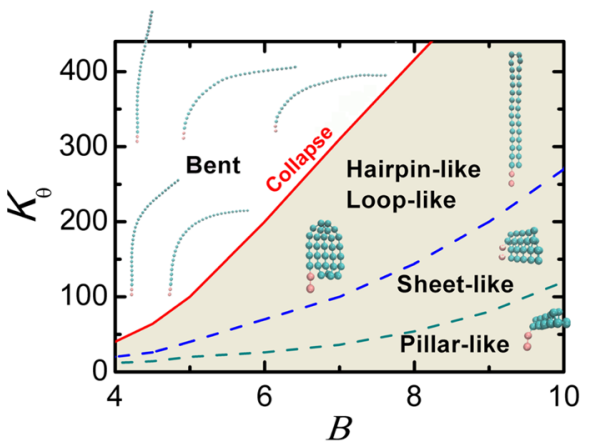

(b)

Figure 2. (a) Typical phase diagram of an anchored magnetic filament. (b) Phase diagram near the collapsing region for filaments with $\xi=1.0$ and $L$ $=30$, which is also marked by the dashed box in (a).

The structural properties of the filaments are determined by the competition between the bonding and dipolar interactions. Note that our system and the choice of the interaction potentials are different from that of Sanchez et al. ${ }^{32,35}$ where they considered ferromagnetic polymers. The bond-dipole coupling effect presents in the ferromagnetic case is absent in the case of superparamagnetic particles, whose magnetic moment can freely rotate without physical rotation of the colloid.

\section{SIMULATION METHOD}

We use coarse-grained MD method to simulate superparamagnetic filaments of different length $L$ and composition $\xi$ in constant external magnetic field $\mathbf{B}=(B, 0,0)$. The conservative force $\mathbf{F}_{c}=-\nabla U$ is derived from the interparticle interactions (eq 4). Since the dipole moments of superparamagnetic particles can freely change direction, we do not consider particle rotation. Since the dipole-dipole interactions favor magnetic beads to line up along the direction of the external field, the straight filament first bends (Figure 1b) and then collapses with the increase of $B$. We implement Langevin thermostat by imposing a friction force $\mathrm{F}_{\mathrm{f}}=-m v / \gamma$ with the damping coefficient $\gamma=0.2$ and a random force $\mathbf{F}_{\mathrm{r}}=\mathbf{R} \sqrt{2 k_{\mathrm{B}} \mathrm{Tm} / \mathrm{d} t \gamma}$ with the components $R_{x, y, z}$ Gaussiandistributed random variables with zero mean and unit variance. The total force $\mathbf{F}$ on each particle is

$$
\mathbf{F}=\mathbf{F}_{\mathrm{c}}+\mathbf{F}_{\mathrm{f}}+\mathbf{F}_{\mathrm{r}}
$$

The length of the time step $\mathrm{d} t$ was chosen so that in a single simulation step the beads move on average a fraction $1 / 20$ of the filament's radius of gyration.

In order to determine stable configurations for filaments with various parameters, we start with a straight filament and implement two simulation methods: simulated annealing and parallel tempering. In annealing, we initially increase the temperature from $T=1$ to $T=30$ (or $T=50$ for very rigid filaments) in $10^{6}$ simulation steps. Then, we slowly quench the system to $T=1$ in $6 \times 10^{8}$ simulation steps. The final configuration is obtained after another $10^{8}$ simulation steps in the NVT ensemble. In parallel tempering, 14 configurations are computed in parallel, with replica temperatures distributed between $T=1$ and $T=60$. The values of the replica temperatures are chosen so that the replica exchange probability is finite even in very strong external field. The stable structures are obtained after approximately $8 \times 10^{8}$ simulation steps. We compute the stable structures at different values of $B$ and $K_{\theta}$ with fixed $K_{\phi}=200, K_{r}=4000$, and $r_{0}=2$ 1/6. We choose to keep $K_{\phi}$ and $K_{r}$ constant because our preliminary runs indicated that the phase behavior of the system is not very sensitive to these two parameters. However, for very small $K_{r} \lesssim 10^{3}$ or very large $K_{\phi} \gtrsim 10^{4}$, the collapsed structures of the filament in strong external field are affected, i.e., they become much less ordered. Note that in our simulations a relatively large value of the stretching rigidity, $K_{r}$, is used, i.e., the connected beads are bound to a very low energy state. Thus, our simulation results are insensitive to the long-range properties of the interbead potential and qualitatively similar results are obtained by replacing the harmonic potential with elasticoplastic interactions such as Morse potential. $^{37}$

\section{RESULT AND DISCUSSION}

A typical phase diagram depicting the shapes of the filaments (Figure 2a) for different bending rigidity $K_{\theta}$ and magnetic field strength $B$ comprises regions of straight, bent, and folded chains. The threshold value of the external field needed for filaments to bend, $B_{\mathrm{b}}\left(K_{\theta}\right)$, is called the bending line, while the collapse line $B=B_{c}\left(K_{\theta}\right)$ separates the bent filaments and folded shapes. In the following we first discuss the collapse line and various shapes observed for filaments with different composition $\xi$ in the collapsed region and then the bending line and bending behavior of the filaments at lower field strengths or higher rigidity.

Collapsing Behavior of Magnetic Filaments: $\xi=1$. Folded shapes of filaments composed of $L=30$ magnetic beads $(\xi=1.0)$ are summarized in Figure $2 \mathrm{~b}$. We observe structures reminiscent of loops, sheets, and compact pillarlike objects. At constant $K_{\theta}$ and upon increasing $B$, the filaments initially fold into a loop or hairpinlike structure after crossing the potential barrier between the bent and collapsed shape. Shorter or stiffer filaments are more likely to fold into a hairpinlike structure than longer or softer ones. We tested how the onset of the collapsing transition depends on the total number of the beads $L$ and observed that the position of the collapse line moves to the right of the phase diagram with decreasing $L$. At larger $B$, the filaments fold into a "double-layered" sheetlike structure and finally into closely packed bulk pillarlike structure. Simulation results of both parallel tempering and slow-quenching indicate that the looplike, sheetlike, and pillarlike structures are stable ones, but they become metastable with respect to random coillike structure when $K_{\theta} \lesssim 40$ and $L<20$. The morphology of the collapsed structures and the typical length scale (e.g., the 
width of the pillar and sheet) depend only very weakly on $L$; see Figure 3. The effect of bond stiffness $K_{r}$ and twist rigidity $K_{\phi}$ on the structural properties is explored in the Supporting Information (see Figure S1).

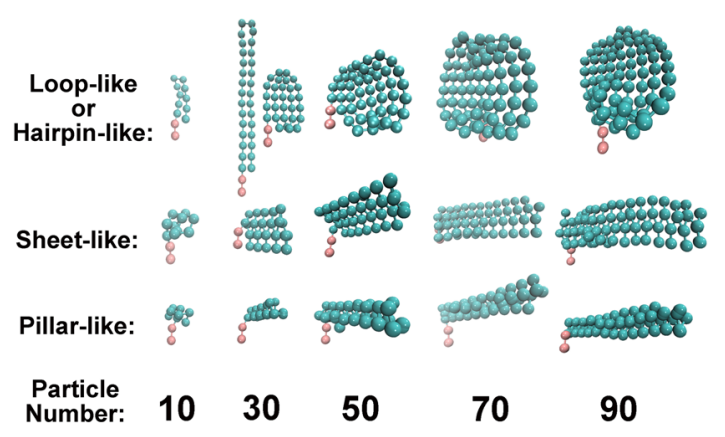

Figure 3. Comparison of collapsed structures for filaments of different particle number $L$ in different regions of the phase diagram.

The folding transition emerges as a consequence of the competition between elastic and magnetic interactions. In order to better understand its mechanism, we studied a simplified model filament consisting of three magnetic beads that interact via the bending and dipole-dipole interactions only $\tilde{U}=\sum_{i<j}$ $U_{i j}^{\mathrm{pp}}+\sum_{i} U_{i}^{\theta}$, thus neglecting the WCA and bond rigidity (the torsion term being constant anyway). We implement the activeset algorithm (see Appendix. A) to numerically determine the minimum energy $\tilde{U}$ of such filaments with fixed bending elasticity $K_{\theta}=10$ in varying external field strength $B$. Figure 4

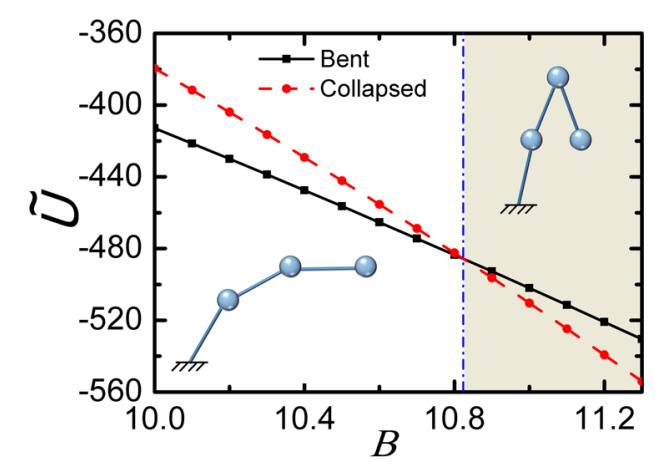

Figure 4. Energy $\tilde{U}$ of an anchored filament consisting of three magnetic beads with $K_{\theta}=10$ at different magnetic field strengths $B$. We compare the energy of a bent filament to the energy of a collapsed structure, both of which are determined by the energy minimization procedure.

shows $\tilde{U}(B)$ for bent and collapsed configurations. Bent configuration is favored for values of $B$ below the threshold $B_{\mathrm{c}} \approx 10.8$, while above the threshold $\left(B>B_{\mathrm{c}}\right)$ the collapsed state wins indicating that the angle of the third bead $\theta_{3}$ tends to be obtuse at large magnetic field magnitudes where the gain in magnetic attraction overcompensates the elastic penalty for extra bending. Such a transition takes place at a smaller threshold $B_{c}$ in the case of longer filaments.

See Movie Mov.1-Mov.4 in the Supporting Information for how the collapse of the exclusive superparamagnetic filaments takes place.

Collapsing Behavior of Neutral-Magnetic Filaments: $\xi<1$. Figure 5 shows phase behavior near the collapsing region for anchored neutral-magnetic combined filament with " $-1-$

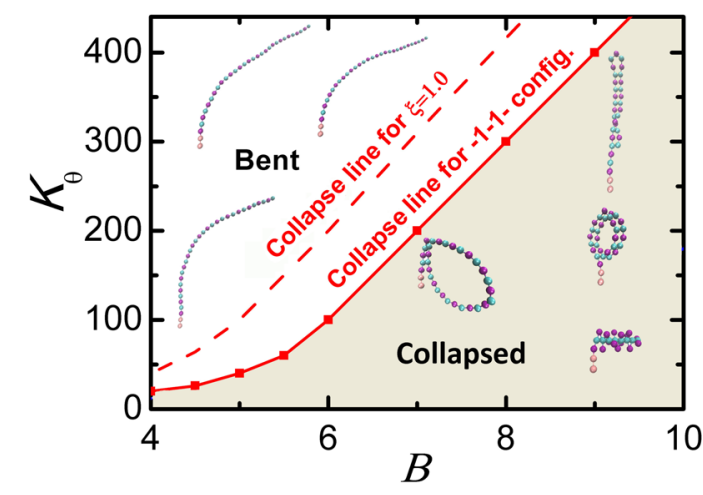

Figure 5. Phase diagram near the collapsing region for anchored neutral-magnetic combined filament of " $-1-1-$ " unit sequence. The blue and red spheres respectively represent the magnetic and neutral beads.

$1-$ " unit sequence $(\xi=0.5)$. Similarly to the case of $\xi=1$ (Figure 2b), the system enters into the collapsed state after crossing the collapse line, which is here shifted toward larger field strengths $B$. By performing parallel tempering simulations, we explore how the equilibrium self-assembled structures depend on the bending rigidity $K_{\theta}$ and the external field $B$. We observe hairpinlike (large $B$, large $K_{\theta}$ ), helicoidal (large $B$, moderate $K_{\theta}$ ), pillarlike (large $B$, small $K_{\theta}$ ), or ringlike (moderate $B$, moderate $K_{\theta}$ ) structures as plotted in Figure 5 .

Generally, for $\xi<1$ the morphology of the observed structures is similar to that of the case $\xi=1$ with 1D (loops), 2D (sheets), and 3D (collapsed clusters) structures. However, the phase behavior is richer and dependent not only on $L$ and $\xi$ but also on the specific sequence $-N_{n}-N_{m}$ - of neutral and magnetic beads within the filament. A complete mapping of the phase behavior is therefore a formidable task, and here we present a glimpse into some of the structures that can be observed. A more thorough study will make sense once a specific experimental realization becomes available so that parameter space can be limited accordingly.

Figure 6 depicts typical simulation snapshots of filaments composed of a mixture of magnetic and neutral colloids with varying $K_{\theta}$ in a strong external field. We observe a clear tendency for phase separation of magnetic and nonmagnetic beads reminiscent of protein folding with the separation of hydrophobic and hydrophilic groups. In our system, we observed ringlike, spoonlike, or racketlike structures with neutral segments either "pinned" together if they are short in length (e.g., " $-1-1-$ " or " $-3-5-$ " sequence with $K_{\theta} \sim 400$ ), or squeezed out from the sticky part if they are long enough (e.g., " $-10-10-$ " sequence with $K_{\theta} \sim 400$ ). The sheets do not fully assemble as was the case in Figure $2 \mathrm{~b}$ for filaments of intermediate bending rigidity. Here, long-enough filaments $\left(N_{\mathrm{m}}\right.$ $\gtrsim 25)$ exhibit only a partial formation of sheetlike structures (see snapshot for "30-30-30" unit sequence with $K_{\theta} \sim 200$ in Figure 6). Finally, for very soft filaments, the collapsed structures are characterized by pillarlike or rodlike clusters of magnetic beads and excluded segments of neutral ones. The dynamics of the collapse of some of these filaments is shown in the movies in the Supporting Information (Mov.5-Mov.7).

We also analyzed the collapsing behavior for filaments with random neutral-magnetic sequences. While for soft filaments the equilibrated structures are more or less the same as that for periodic sequences (i.e., the magnetic beads are collapsed and buried inside the cluster, while the neutral ones are expelled) 


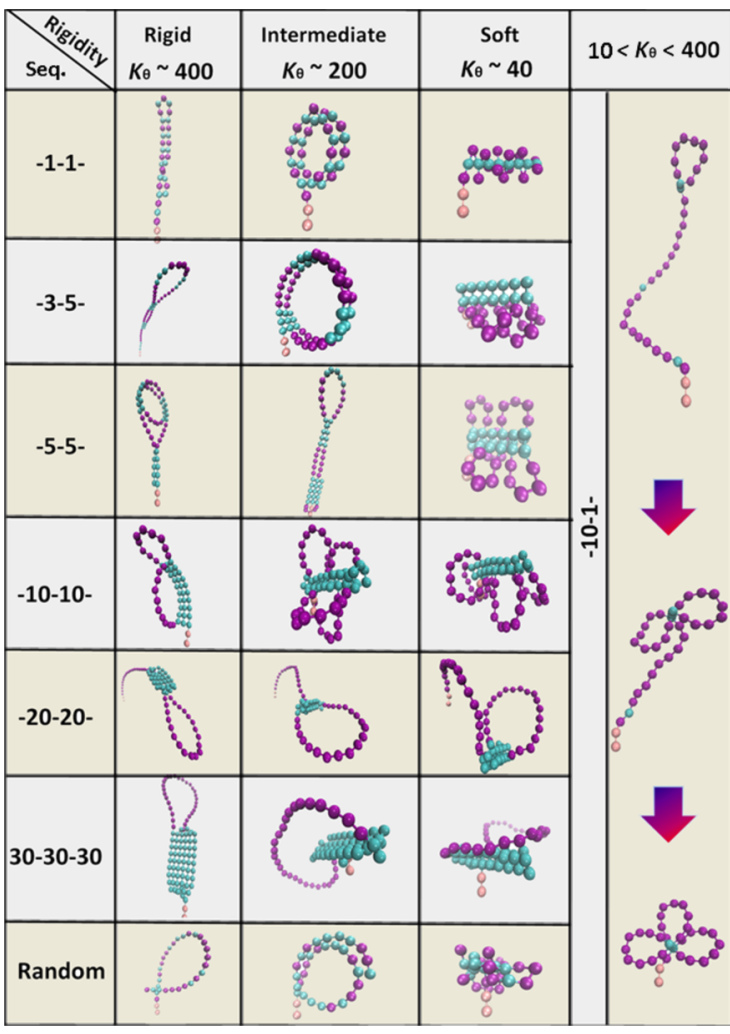

Figure 6. Simulation snapshots of filaments composed of neutral and magnetic beads. The first three columns depict the structures formed by the filaments with different bending rigidity $K_{\theta}$ in constant magnetic field $B=9$. Filaments with different periodic sequence of magnetic (blue) and neutral (red) beads, " $-N_{\mathrm{n}}-N_{\mathrm{m}}-$ ", are placed in different rows. In the last row, the "30-30-30" sequence denotes a filament of 60 magnetic particles and 30 neutral ones. In the rightmost column, the kinetics of the collapsing process for the "-10-1-" filament with a total number of three magnetic particles is presented.

but less ordered, for rigid and semirigid filaments we only observe ringlike or helicoidal structures (similar to that plotted in Figure 5 for filament of " $-1-1-$ " unit sequence). The absence of the other structures observed in the periodic sequences can be explained by the high energy penalty for the segments with incommensurate lengths to form the structures with underlying periodicity. Although unbonded short magnetic-beads-rich segments can be "pinned" together by magnetic attractions to form a ring, it is very unlikely to closely stick long segments of very different sequences together. For instance, hairpinlike structure is stable for rigid filament of "1-1-" unit sequence, but definitely unstable for random sequences. In the latter case, it is very unlikely for magnetic beads to find their "pairs" on the other leg of the hairpin, so that the internal energy remains high.

Bending. We perform numerical energy minimization (see Appendix A) in order to determine the position of the bending line. The result is presented in Figure 7a for the case of $\xi=1$. As expected, we observe that the bending line shifts to the left with increasing number of beads $L$. According to the magnetoelastic theory, ${ }^{13}$ the change from straight to bent state of the superparamagnetic filament is actually a Landau second-order transition. Indeed, if we plot $K_{\theta}^{1 / 2} / B_{\mathrm{b}}$ (where $B_{\mathrm{b}}$ is the threshold value of bending magnetic flux density) as a function of $L$ (Figure $7 \mathrm{~b}$ ), the results collapse onto a single line, which denotes that $B_{\mathrm{b}}$ depends linearly on $1 / L$ and $K_{\theta}^{1 / 2}$, in accord with predictions in ref 13 .

At fixed $K_{\theta}$, with the increase of $B$ the system crosses the bending line and enters into the bending region. Starting from the simplest cases, we first analyze the bending behavior for filaments with three magnetic particles (the even simpler case of two beads is discussed in detail in the Supporting Information); Figure $8 \mathrm{~b}$ compares $\theta$ for an anchored filament consisting of three superparamagnetic particles at $B=5$. With the increase of $K_{\theta}$, all the three bending angles decrease when $K_{\theta}>200$, but the dangling angle $\theta_{3}$ decreases less rapidly than $\theta_{2}\left(\right.$ or $\left.\theta_{1}\right)$. We find a good agreement between the MD and EM results. Generally, we observe that the angles $\theta_{1}$ and $\theta_{2}$ are the same. This follows naturally from the energy minimization, where the two quantities appear symmetrically.

The threshold value of bending magnetic flux density $B_{\mathrm{b}}$ can be easily read off the $\theta-B$ relation in Figure $8 \mathrm{c}: B_{\mathrm{b}}=2.625$ for $K_{\theta}=200$ and $B_{\mathrm{b}}=3.106$ for $K_{\theta}=280$. We further observe that the value of $\theta_{2}$ (or $\theta_{1}$ ) monotonically increases with $B$ and decreases with $K_{\theta}$, while the value of the dangling angle $\theta_{3}$ is nonmonotonic.

Here we note that the decrease of $\theta_{3}$ with the decrease of $K_{\theta}$ in Figure $8 b$ and the increase of $B$ in Figure $8 c$ is not due to the presence of dipole-dipole attraction between the first and the third beads, but rather the increase of $\theta_{1}$ and $\theta_{2}$ (within the bent phase the equilibrium structure would always entail $\theta_{1}+\theta_{2}$ $\left.+\theta_{3} \leq \pi / 2\right)$. This can be validated by calculating the bending angles for system that neglects dipole-dipole interaction between the first and the third beads (eq 11 in Appendix A). As illustrated in Figure 8b, we find that the bending angles for the system without dipole-dipole interaction between the first and the third beads (blue dotted lines) are very close to those

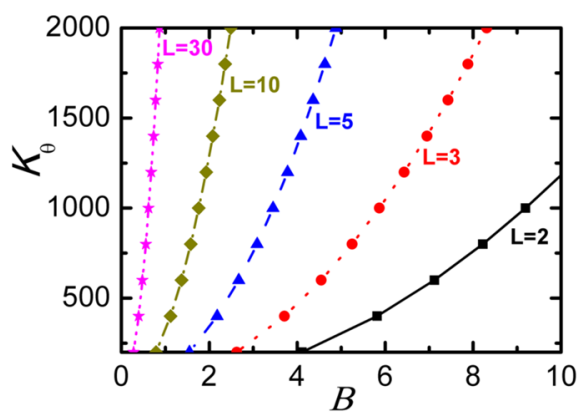

(a)

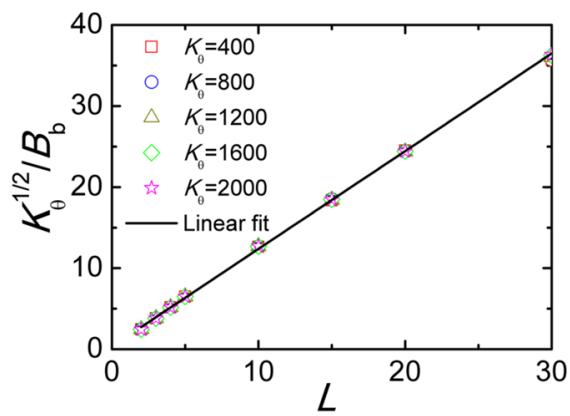

(b)

Figure 7. (a) Dependence of the bending line on the total number of the beads $L$, for exclusive magnetic filament. (b) Linear fit of $K_{\theta}^{1 / 2} / B_{\mathrm{b}}-L$ relation. 


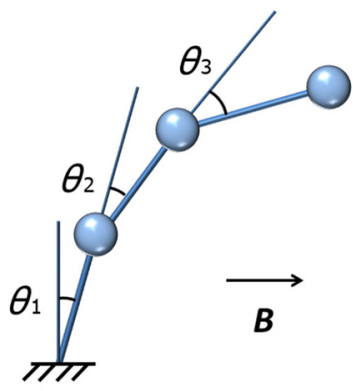

(a)

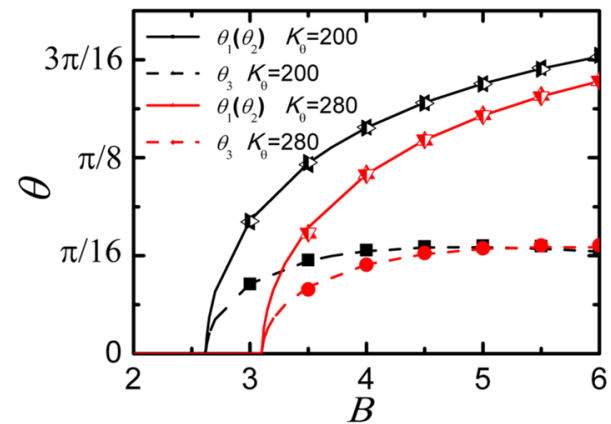

(c)

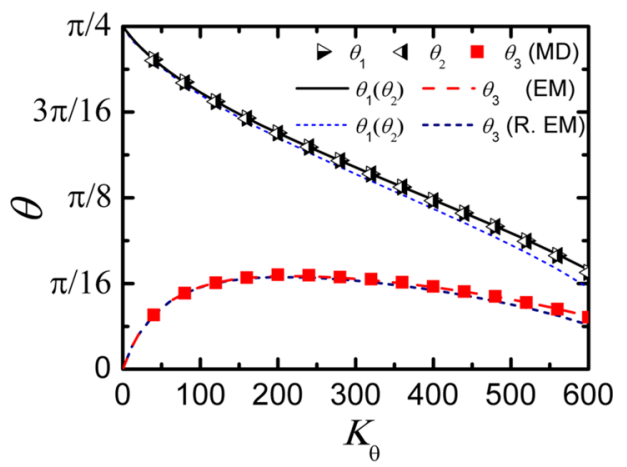

(b)

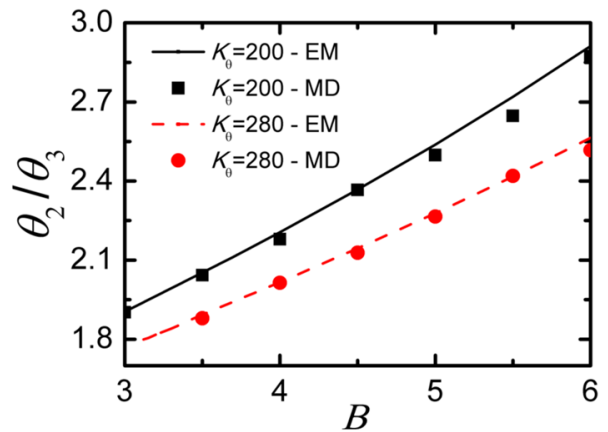

(d)

Figure 8. (a) Sketch of bending angles for filament consists of three particles. (b) $\theta-K_{\theta}$ relation at $B=5$, (c) $\theta-B$ relation at $K_{\theta}=200$ and $K_{\theta}=280$, and (d) $\theta_{2} / \theta_{3}-B$ relation at $K_{\theta}=200, K_{\theta}=240$, and $K_{\theta}=280$, for anchored filament consisting of three superparamagnetic particles.

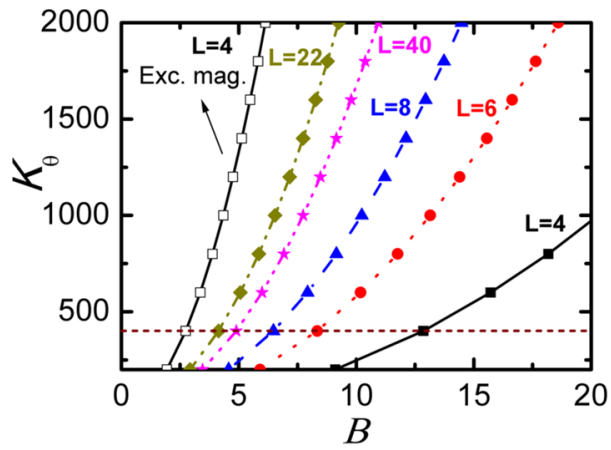

(a)

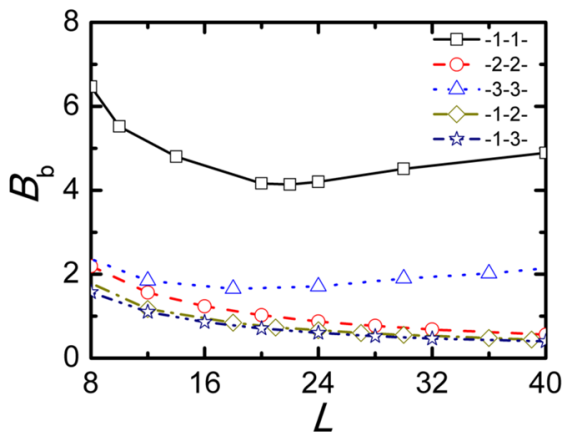

(b)

Figure 9. (a) Dependence of the bending line on the total particle number $L$, for neutral-magnetic filament with a " $-1-1-$ " sequence. The horizontal dashed line links the value of $B_{\mathrm{b}}$ for filaments of different $L$ at $K_{\theta}=400$. (b) $B_{\mathrm{b}}-L$ relation at $K_{\theta}=400$ for neutral-magnetic filament of different sequences as indicated in the legend.

for the system that considers next-neighbor magnetic interaction (black solid and red dashed lines) at fixed $K_{\theta}$, especially when $K_{\theta}$ is small. The values of $\theta_{3}$ and $\theta_{1}\left(\theta_{2}\right)$ can be extrapolated to 0 and $\pi / 4$ respectively for $K_{\theta}=0$.

Both simulation and theoretical optimization show that at constant $B$, the ratio of the angles $\theta_{2} / \theta_{3}$ is larger for smaller $K_{\theta}$; see Figure $8 \mathrm{~d}$. We may therefore predict that the larger the value of $K_{\theta}$, the more continuous the bending of the filaments becomes. In Figure $8 \mathrm{~d}$ the energy minimization results are slightly above the simulations, which is due to the fact that in MD simulations the bonds between particles are slightly stretched, which increases the value of $\theta_{3}$.

Figure 9a presents how the position of bending line depends on the total particle number, $L$, for neutral-magnetic filament with "-1-1-" unit. We find that adding neutral particles between magnetic ones strongly increases the value of $B_{\mathrm{b}}$ at constant $K_{\theta}$. For instance, as illustrated in Figure 9a, the position of the bending line for " $-1-1-$ " unit filament with $L$ $=4$ is quite different from that for purely magnetic filament with $L=4$.

The $B_{\mathrm{b}}-L$ relation for neutral-magnetic filament of different unit sequence at constant $K_{\theta}=400$ is shown in Figure 9b. We find that, at fixed $L$, the bending threshold depends not only on magnetic fraction $\xi$ but also on the sequence of the magnetic particles within the filaments. Moreover, we observe that the $B_{\mathrm{b}}-L$ relation even becomes nonmonotonic for neutralmagnetic filament of “ $-1-1-$ ” and “-3-3-" sequences, as illustrated in Figure 9b.

\section{CONCLUSION}

We have explored the folding properties of anchored magnetoelastic filaments in a wide range of parameters such 
as bending rigidity, external magnetic field strength, and the filament composition. We observed a rich cohort of collapsed structures including loops, sheets, and helicoidal assemblies. Since it is experimentally relatively straightforward to control the structure formation by applying external field, or by controlling the filaments composition, our results may prove useful in designing applications in bioengineering or design of new materials with complex building blocks. As an example, filaments of spoonlike or ringlike structure might be utilized as a microscopic lasso to grab and transport nanoparticles or macromolecules.

\section{APPENDIX. A ENERGY MINIMIZATION}

We use active-set algorithm ${ }^{38,39}$ to find the minimum of nonlinear multivariable function of internal energy. We first analyze the internal energy of filament that consists of only two magnetic particles. For simplicity, we assume that the distance between the particles is fixed, and the filament is fixed in a $2 \mathrm{D}$ plane. In addition, we assume the value of the dipole moment, $\overrightarrow{\mathbf{p}}$, of each particle equals the external field, and there is no Lennard-Jones interactions. Thus, the selected internal energy of the system, $\tilde{U}$, can be written as

$$
\tilde{U}=\frac{1}{2} K_{\theta} \theta_{1}{ }^{2}+\frac{1}{2} K_{\theta} \theta_{2}{ }^{2}+\frac{p^{2}-3 p^{2} \sin ^{2}\left(\theta_{1}+\theta_{2}\right)}{r^{3}}
$$

where $\theta_{1}$ and $\theta_{2}$ are the bending angles of the two particles. Taking a look at the equation, we note that here $\tilde{U}$ always reaches its minimum when $\theta_{1}=\theta_{2}$. We therefore assume $\theta_{1}=$ $\theta_{2}=\theta$, and eq 6 reduces to

$$
\tilde{U}=K_{\theta} \theta^{2}+\frac{p^{2}-3 p^{2} \sin ^{2}(2 \theta)}{r^{3}}
$$

We next analyze the internal energy of filament that consists of three magnetic particles. The selected internal energy of this system is written as

$$
\begin{gathered}
\tilde{U}=\frac{1}{2} K_{\theta} \theta_{1}{ }^{2}+\frac{1}{2} K_{\theta} \theta_{2}{ }^{2}+\frac{1}{2} K_{\theta} \theta_{3}^{2} \\
+\frac{2 p^{2}-3 p^{2} \sin ^{2}\left(\theta_{1}+\theta_{2}\right)-3 p^{2} \sin ^{2}\left(\theta_{1}+\theta_{2}+\theta_{3}\right)}{r^{3}} \\
+\frac{p^{2}-3 p^{2} \sin ^{2}\left(\theta_{1}+\theta_{2}+\frac{1}{2} \theta_{3}\right)}{r_{13}{ }^{3}},
\end{gathered}
$$

where $\theta_{1}, \theta_{2}$, and $\theta_{3}$ represent the bending angles of the three particles, and $r_{13}$ gives the distance between the first and the last particle, which is written as

$$
r_{13}=\sqrt{2 r^{2}-2 r^{2} \cos \left(\pi-\theta_{3}\right)}
$$

Still we can find in eq 8 that $\theta_{1}$ and $\theta_{2}$ are interchangeable, and $\tilde{U}$ is always smaller when they are equal. Thus, we assume $\theta_{1}=\theta_{2}$, and eq 8 reduces to

$$
\begin{gathered}
\tilde{U}=K_{\theta} \theta_{2}{ }^{2}+\frac{1}{2} K_{\theta} \theta_{3}{ }^{2} \\
+\frac{2 p^{2}-3 p^{2} \sin ^{2}\left(2 \theta_{2}\right)-3 p^{2} \sin ^{2}\left(2 \theta_{2}+\theta_{3}\right)}{r^{3}} \\
+\frac{p^{2}-3 p^{2} \sin ^{2}\left(2 \theta_{2}+\frac{1}{2} \theta_{3}\right)}{r_{13}{ }^{3}} .
\end{gathered}
$$

Note that eq 10 is applicable even when $\theta_{3}>\pi / 2$, i.e., when the filament collapses. We can compare the energy of bent and collapsed states by setting the boundary condition for $\theta_{3}$ as $\theta_{3}$ $\in[0, \pi / 2]$ and $\theta_{3} \in[\pi / 2, \pi]$, respectively.

Since $r_{13}$ depends only negatively on $\theta_{3}$, the dipole-dipole interaction between the first and the third beads (the last term in eq 10) would always favor larger value of $\theta_{3}$. If such interaction is not taken into account, we have

$$
\begin{aligned}
\tilde{U}= & K_{\theta} \theta_{2}{ }^{2}+\frac{1}{2} K_{\theta} \theta_{3}{ }^{2} \\
& +\frac{2 p^{2}-3 p^{2} \sin ^{2}\left(2 \theta_{2}\right)-3 p^{2} \sin ^{2}\left(2 \theta_{2}+\theta_{3}\right)}{r^{3}}
\end{aligned}
$$

\section{ASSOCIATED CONTENT}

\section{S Supporting Information}

The Supporting Information is available free of charge on the ACS Publications website at DOI: 10.1021/acs.langmuir.6b02268.

Study of ssembly of superparamagnetic filaments in external field (PDF)

Magnetic filament self-assembles into a hairpinlike structure (AVI)

Magnetic filament self-assembles into a looplike structure (AVI)

Magnetic filament self-assembles into a sheetlike structure. (AVI)

Magnetic filament self-assembles into a pillarlike structure (AVI)

Neutral-magnetic filament self-assemblesinto a ringlike structure (AVI)

Neutral-magnetic filament self-assembles into a pillarlike structure (AVI)

Collapsing process of a neutral-magnetic filament of “10-1-" sequence (AVI)

\section{AUTHOR INFORMATION}

\section{Corresponding Authors}

*E-mail:weijiachen@lnm.imech.ac.cn.

*E-mail:jd489@cam.ac.uk.

\section{Notes}

The authors declare no competing financial interest.

\section{ACKNOWLEDGMENTS}

We acknowledge insightful discussions with the participants of the 2016 CECAM workshop "Emergent dynamics of out-ofequilibrium colloidal systems at nano- to microscales", especially with Ning Wu, Anand Yethiraj, Qian Chen, Kyongok Kang, and Tomaz Mohoric. The work was supported by the EC grant NANOTRANS 674979 MSC ETN, Fundamental Research Funds for the Central Universities of P. R. China under the project buctrc201422 and the National Natural Science Foundations of China (Grants No.11232013 and No.11472285).

\section{REFERENCES}

(1) Das, S.; Banik, M.; Chen, G.; Sinha, S.; Mukherjee, R. Polyelectrolyte brushes: Theory, modelling, synthesis, and applications. Soft Matter 2015, 11, 8550-8583.

(2) Liu, F.; Urban, M. W. Recent advances and challenges in designing stimuli-responsive polymers. Prog. Polym. Sci. 2010, 35, 323. 
(3) Minko, S. Responsive polymer brushes. J. Macromol. Sci., Polym. Rev. 2006, 46, 397-420.

(4) Stuart, M. A. C.; Huck, W. T.; Genzer, J.; Muller, M.; Ober, C.; Stamm, M.; Sukhorukov, G. B.; Szleifer, I.; Tsukruk, V. V.; Urban, M.; Winnik, F.; Zauscher, S.; Luzinov, I.; Minko, S. Emerging applications of stimuli-responsive polymer materials. Nat. Mater. 2010, 9, 101-113.

(5) Curk, T.; Martinez-Veracoechea, F. J.; Frenkel, D.; Dobnikar, J. Collective ordering of colloids in grafted polymer layers. Soft Matter 2013, 9, 5565.

(6) Curk, T.; Martinez-Veracoechea, F. J.; Frenkel, D.; Dobnikar, J. Nanoparticle organization in sandwiched polymer brushes. Nano Lett. 2014, 14, 2617-2622.

(7) Liu, Y. H.; Chew, L. Y.; Yu, M. Y. Self-assembly of complex structures in a two-dimensional system with competing interaction forces. Phys. Rev. E 2008, 78, 066405.

(8) Lu, P. J.; Conrad, J. C.; Wyss, H. M.; Schofield, A. B.; Weitz, D. A. Fluids of clusters in attractive colloids. Phys. Rev. Lett. 2006, 96, 028306.

(9) Reichhardt, C. O.; Reichhardt, C.; Bishop, A. R. Structural transitions, melting, and intermediate phases for stripe-and clumpforming systems. Phys. Rev. E 2010, 82, 041502.

(10) Hergt, R; Hiergeist, R.; Zeisberger, M.; Glockl, G.; Weitschies, W.; Ramirez, L. P.; Hilger, I.; Kaiser, W. A. Enhancement of AC-losses of magnetic nanoparticles for heating applications. J. Magn. Magn. Mater. 2004, 280, 358-368.

(11) Li, K. H.; Yellen, B. B. Magnetically tunable self-assembly of colloidal rings. Appl. Phys. Lett. 2010, 97, 083105.

(12) Fayol, D.; Frasca, G.; Le Visage, C.; Gazeau, F.; Luciani, N.; Wilhelm, C. Use of magnetic forces to promote stem cell aggregation during differentiation and cartilage tissue modeling. Adv. Mater. 2013, $25,2611-2616$.

(13) Gerbal, F.; Wang, Y.; Lyonnet, F.; Bacri, J.-C.; Hocquet, T.; Devaud, M. A refined theory of magnetoelastic buckling matches experiments with ferromagnetic and superparamagnetic rods. Proc. Natl. Acad. Sci. U. S. A. 2015, 112, 7135-7140.

(14) Dobnikar, J.; Snezhko, A.; Yethiraj, A. Emergent colloidal dynamics in electromagnetic fields. Soft Matter 2013, 9, 3693-3704.

(15) Muller, K.; Osterman, N.; Babic, D.; Likos, C. N.; Dobnikar, J.; Nikoubashman, A. Pattern formation and coarse-graining in twodimensional colloids driven by multiaxial magnetic fields. Langmuir 2014, 30, 5088-5096.

(16) Benkoski, J. J.; Breidenich, J. L.; Uy, O. M.; Hayes, A. T.; Deacon, R. M.; Land, H. B.; Spicer, J. M.; Keng, P. Y.; Pyun, J. Dipolar organization and magnetic actuation of flagella-like nanoparticle assemblies. J. Mater. Chem. 2011, 21, 7314.

(17) Townsend, J.; Burtovyy, R.; Galabura, Y.; Luzinov, I. Flexible chains of ferromagnetic nanoparticles. ACS Nano 2014, 8, 6970-6978.

(18) Cebers, A.; Erglis, K. Flexible magnetic filaments and their applications. Adv. Funct. Mater. 2016, 26, 3783.

(19) Cebers, A. Dynamics of a chain of magnetic particles connected with elastic linkers. J. Phys.: Condens. Matter 2003, 15, S1335.

(20) Shcherbakov, V. P.; Winklhofer, M. Bending of magnetic filaments under a magnetic field. Phys. Rev. E 2004, 70, 061803.

(21) Cebers, A.; Javaitis, I. Bending of flexible magnetic rods. Phys. Rev. E 2004, 70, 021404.

(22) Cebers, A. Flexible magnetic filaments. Curr. Opin. Colloid Interface Sci. 2005, 10, 167-175.

(23) Cebers, A.; Cirulis, T. Magnetic elastica. Phys. Rev. E 2007, 76, 031504.

(24) Belovs, M.; Cebers, A. Nonlinear dynamics of semiflexible magnetic filaments in an ac magnetic field. Phys. Rev. E 2006, 73, 051503.

(25) Erglis, K.; Zhulenkovs, D.; Sharipo, A.; Cebers, A. Elastic properties of DNA linked flexible magnetic filaments. J. Phys.: Condens. Matter 2008, 20, 204107.

(26) Dreyfus, R.; Baudry, J.; Roper, M. L.; Fermigier, M.; Stone, H. A.; Bibette, J. Microscopic artificial swimmers. Nature 2005, 437, 862865.
(27) Gauger, E.; Stark, H. Numerical study of a microscopic artificial swimmer. Phys. Rev. E 2006, 74, 021907.

(28) Roper, M.; Dreyfus, R.; Baudry, J.; Fermigier, M.; Bibette, J.; Stone, H. A. On the dynamics of magnetically driven elastic filaments. J. Fluid Mech. 2006, 554, 167-190.

(29) Osterman, N.; Poberaj, I.; Dobnikar, J.; Frenkel, D.; Ziherl, P.; Babic, D. Field-induced self-assembly of suspended colloidal membranes. Phys. Rev. Lett. 2009, 103, 228301.

(30) Pak, O. S.; Gao, W.; Wang, J.; Lauga, E. High-speed propulsion of flexible nanowire motors: Theory and experiments. Soft Matter 2011, 7, 8169-8181.

(31) Philippova, O.; Barabanova, A.; Molchanov, V.; Khokhlov, A. Magnetic polymer beads: Recent trends and developments in synthetic design and applications. Eur. Polym. J. 2011, 47, 542-559.

(32) Sanchez, P. A.; Pyanzina, E. S.; Novak, E. V.; Cerda, J. J.; Sintes, T.; Kantorovich, S. S. Supramolecular magnetic brushes: The impact of dipolar interactions on the equilibrium structure. Macromolecules 2015, 48, 7658-7669.

(33) Evans, B. A.; Shields, A. R.; Carroll, R. L.; Washburn, S.; Falvo, M. R.; Superfine, R. Magnetically actuated nanorod arrays as biomimetic cilia. Nano Lett. 2007, 7, 1428-1434.

(34) Erglis, K.; Livanovics, R.; Cebers, A. Three dimensional dynamics of ferromagnetic swimmer. J. Magn. Magn. Mater. 2011, 323, 1278-1282.

(35) Sanchez, P. A.; Pyanzina, E. S.; Novak, E. V.; Cerda, J. J.; Sintes, T.; Kantorovich, S. S. Magnetic filament brushes: Tuning the properties of a magnetoresponsive supracolloidal coating. Faraday Discuss. 2016, 186.24110.1039/C5FD00133A.

(36) Rapaport, D. C. The Art of Molecular Dynamics Simulation, 2nd ed.; Cambridge University Press: Cambridge, 2004.

(37) Tanimura, T. Fifth-order two-dimensional vibrational spectroscopy of a Morse potential system in condensed phases. Chem. Phys. 1998, 233, 217-229.

(38) Fletcher, R; Powell, M. A rapidly convergent descent method for minimization. Comput. J. 1963, 6, 163-168.

(39) Goldfarb, D. A family of variable-metric methods derived by variational means. Math. Comput 1970, 24, 23-26. 